\title{
Design and Research on tower escape apparatus based on the principle of escapement mechanism
}

\author{
Kaibao Wang ${ }^{1,2, a}$, Sicheng Qin ${ }^{1, b}$, Kemin Liu ${ }^{2, c}$ and Xinglong Chen ${ }^{2, d}$ \\ ${ }^{1}$ College of Mechanical Science and Engineering, Jilin University, Changchun, Jilin, 130025, China \\ ${ }^{2}$ College of Mechanical Engineering, Beihua University, Jilin, Jilin, 132021, China \\ awangkaibao@126.com, bqinsc@jlu.edu.cn, cliu.kemin@163.com, d502162582@qq.com
}

\section{Keywords: Principle Of Escapement Mechanism, Tower Escape Apparatus, Mechanism Design.}

\begin{abstract}
The tower circular escape apparatus based on the principle of escapement mechanism is introduced in the paper. The emergency evacuation device for fire trapped workers can be used in the place of frequent fire, hotels, office buildings, high-rise residential and stores, etc. The velocity of the principal axis can be controlled automatically by the escapement mechanism. The driving force of the escape apparatus comes from the gravity of the trapped workers. The experiment results show that the velocity of the device can be controlled on the operating range. The symmetrical structure can make more persons with different weight escape alternately and the velocity of descend approximate. In the research, the virtual prototype technology was used to analyse the kinematic characteristics of the mechanical system. The feasibility of the scheme design of mechanical system was proved with the simulation analysis results. The equipment has advantages of the structure is compact, safe and reliable, easy to use. The research of tower circular escape apparatus based on the principle of escapement mechanism can be regarded as the reference of the related research.
\end{abstract}

\section{Introduction}

With the rapid development of China's economy, more and more high-rise buildings was built to meet the needs of the comfortable life. In recent years, the tower fire caused by the lack of fire fighting facilities happened frequently. How to escape from tall building in case of a fire has become the serious social problem. The fire rescue facilities and technology are relatively mature in the developed countries[1-3]. Compared with the foreign countries, the fire rescue facilities and technology used in the tall buildings are relatively backward in our country. Many trapped workers lose there precious life because they are lack of timely assistance. The escape apparatus that can help fire trapped workers to escape from tall building timely, valid, and conveniently was needed.

The tower escape apparatus based on the principle of escapement mechanism researched in this paper is a kind of escape device can help the fire trapped workers to escape from the tall building timely. The device that can be operated by personnel is installed in the balcony or public channel window place. The escape instruments have good performance, carrying capacity, long time work. The driving force of the escape apparatus comes from the gravity of the trapped workers without external energy. The escape device help the fire trapped workers to establish the security beliefs, and provide security for smooth landing.

\section{General structure and the main design index}

Prototype structure and working principle. The tower circular escape apparatus based on the principle of escapement mechanism was comprised by four parts, hold-down mechanism, double escapement mechanism, starting switch and flywheel institutions. The driving force of the escape apparatus comes from the gravity of the trapped workers. With the escapement mechanism and the rotation inertia of flywheel, the intermittent movement was realized. The system structure of the tower escape apparatus is shown in Fig. 1. 


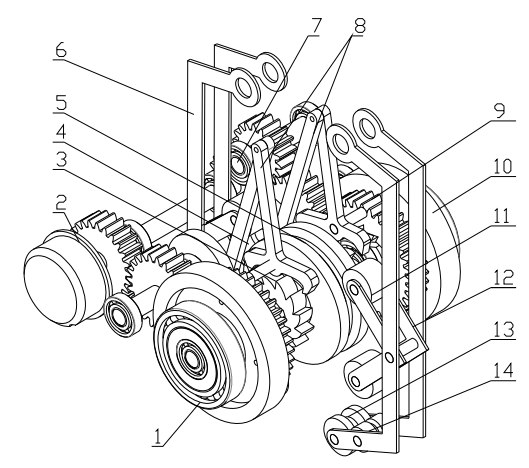

Fig. 1 The system structure of the tower circular escape apparatus

The start switch should be pulled to start the device. The crank and rocker mechanism get the driving force and make the device operation. The escapement claws connected with rocker released the escapement wheel. The escapement wheel and principal axis rotated together and then the trapped workers will landing. The principal axis will stop turning when the rolling wheel turned $120^{\circ}$ that the escapement wheel and escapement claws was fixed. The transmission shaft will drive crank rotate with the influence of flywheel inertia. The escapement claws released the escapement wheel again and the trapped workers will fall again. The series of movement in circulation finally realize the trapped workers landed safely.

Prototype design index. In the design process, the structure sizes have an important impact on the performance of the apparatus. The design index of the tower circular escape apparatus is shown in table 1.

Table 1 The design index of the tower circular escape apparatus

\begin{tabular}{|c|c|c|c|}
\hline Item & Parameters & Item & Parameters \\
\hline The size $/ \mathrm{mm}$ & $300 \times 238 \times 412$ & The strength of machine $/ \mathrm{N}$ & $>4200$ \\
\hline The decreasing speed $(\mathrm{m} / \mathrm{s})$ & $1 \sim 2$ & Height range $/ \mathrm{m}$ & $6 \sim 150$ \\
\hline Weight range $/ \mathrm{kg}$ & $\leq 100$ & The weight $/ \mathrm{kg}$ & $\leq 10$ \\
\hline
\end{tabular}

\section{Analysis and calculation of the escapement speed control mechanism}

The escapement speed control mechanism is the key mechanism of the tower circular escape apparatus based on the principle of escapement mechanism. The speed control of the device can realize through the comprehensive function of two aspects: the storage and release of the flywheel system energy; the combination and separate of the escapement wheel and escapement claws. The velocity of the escapement device is directly related to the gravity of the trapped workers. In the design process, the detailed analysis of calculation of the escapement speed control mechanism was completed in order to determine the functional relationship of the safety speed and the gravity of the trapped workers. We supposed the altitude intercept between the trapped workers and ground is $h$ in the single escapement movement process. According to the dynamics common laws and the function relationship of the related motion parameters, we can know:

$$
\frac{1}{2} J_{1} \omega_{1}^{2}+\frac{1}{2} J_{2} \omega_{2}^{2}+\frac{1}{2} J_{3} \omega_{3}^{2}+\frac{1}{2} J_{4} \omega_{4}^{2}+\frac{1}{2} J_{5} \omega_{5}^{2} \times 2+\frac{1}{2} J_{6} \omega_{6}^{2}+\frac{1}{2} J_{7} \omega_{7}^{2}+\frac{1}{2} J_{8} \omega_{8}^{2}+\frac{1}{2} m v^{2}=m g h
$$

Simultaneous equations:

$$
\left\{\begin{array} { l } 
{ \omega _ { 2 } = \omega _ { 1 } = \omega _ { 4 } } \\
{ \omega _ { 2 } r _ { 2 } = \omega _ { 3 } r _ { 3 } = \omega _ { 4 } r _ { 4 } } \\
{ \omega _ { 5 } = \omega _ { 6 } = \omega _ { 7 } = \omega _ { 1 } }
\end{array} \quad \left\{\begin{array}{l}
\omega_{8} r_{8}=\omega_{6} r_{6} \\
\omega_{8}=\omega_{1} r_{6} / r_{8} \\
J=m r^{2} / 2
\end{array}\right.\right.
$$

According to the theory formula can be concluded that the function relationship of the security speed and the weight of the trapped workers for:

$$
v=\sqrt{A /(c / m+B)} \mathrm{m} / \mathrm{s}
$$


The formula is a nonlinear relationship, $A=5.23 \times 10^{-3} \mathrm{~m}^{3} / \mathrm{s}^{2}, B=2.5 \times 10^{-3} \mathrm{~m}^{2}, C=5.97 \times 10^{-3} \mathrm{~kg} \cdot \mathrm{m}^{2}$.

\section{The main structure design}

The Double Escapement Mechanism Design. The double escapement mechanism composed by escapement wheel, escapement claws and crank and rocker mechanism. The mechanism realized the controlled-release adjustment of the descend velocity. The mechanism has a advantages of the speed cycle stability. The descend velocity can be controlled in the safety range with the double escapement mechanism. The escapement mechanism belongs to the one-way intermittent movement mechanism. Capture means lock the movement of the escapement wheel. Release means clear the movement of the escapement wheel under the action of the flywheel system energy. The flywheel system can store energy again with the operation of the mechanism in order to the continuous operation of device. The structure of double escapement mechanism is shown in Fig. 2.

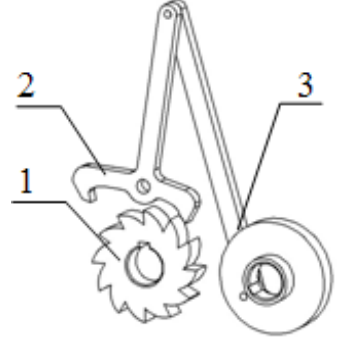

Fig. 2 Structure of escapement mechanism

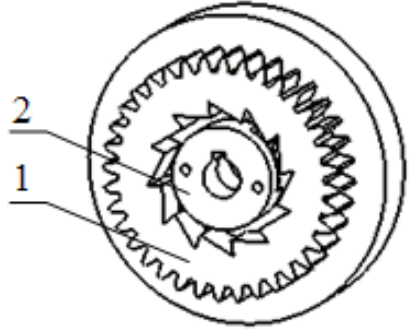

Fig. 3 Structure of flywheel mechanism

The Flywheel Mechanism Design. The flywheel mechanism composed by the flywheel with spokes. The flywheel mechanism that installed in the both sides of the escapement mechanism connect with the crank and rocker mechanism through the drive shaft. The drive force that used to maintain the normal work of the escapement mechanism system transmit to the flywheel mechanism through the external gear ratchet wheel mechanism. The escapement wheel that lock the movement by the escapement claw will rotate again under the influence of the flywheel inertial force. The rotation of the flywheel mechanism ensure the trapped workers can descent to the ground smoothly. The structure of flywheel mechanism is shown in Fig. 3.

The Hold-down Mechanism Design. The hold-down mechanism composed by V rolling frame, rolling wheel, guide wheel and clamp wheel. V rolling frame installed in the middle of the retainer. The guide wheel and clamp wheel installed on the bottom of the retainer should form a certain angle. The wire rope twine around the outside of the guide wheel will pressed in the geneva wheel round rolling frame. The wire rope with the inside torque by the guide wheel will happen between the pure rolling with rolling wheel. The structure of hold-down mechanism is shown in Fig. 4.

\section{Dynamics simulation of The tower circular escape apparatus}

In order to verify the relationship between the release velocity of the escapement mechanism and the weight of the trapped workers, the virtual prototyping model of the escapement mechanism was established using the virtual prototype software ADAMS[4]. The simulation model of the escapement mechanism is shown in Fig. 5. Simulation environment set: model material is steel that the density is $7.85 \times 10^{-6} \mathrm{~kg} / \mathrm{m}^{3}$; the driving force applied range is $200 \mathrm{~N} \sim 1000 \mathrm{~N}$. We regard the velocity of the geneva wheel as the descending velocity of the trapped workers. The simulation curve of the average velocity is shown in Fig. 6.
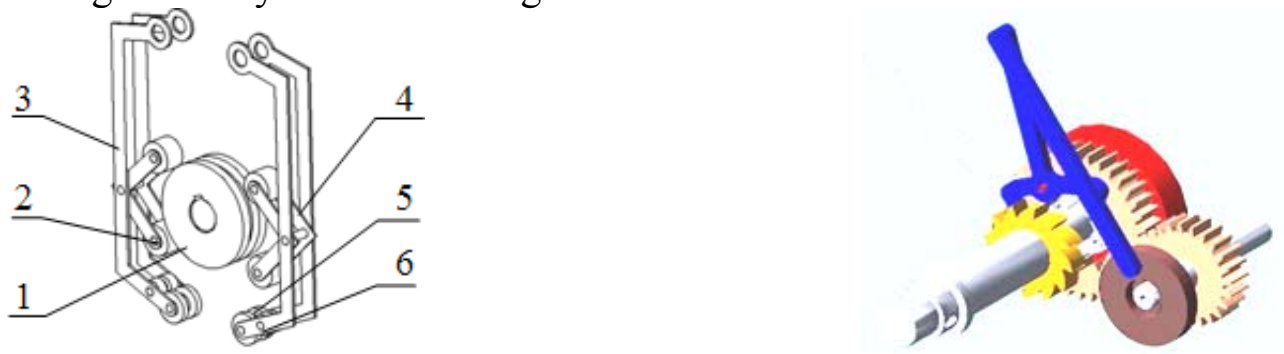
Fig. 4 Structure of hold-down mechanism Fig. 5 Simulation model of escapement mechanism

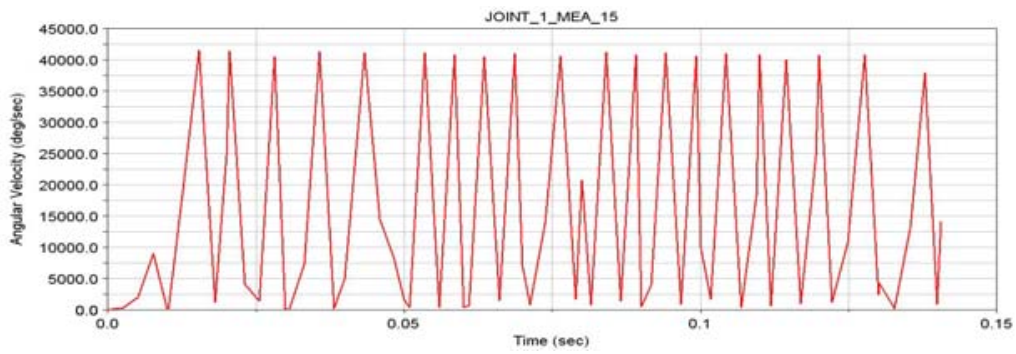

Fig. 6 The simulation curve of the average velocity (the driving forces is $800 \mathrm{~N}$ )

The simulation curve of average velocity of escapement velocity control mechanism when the driving force is $800 \mathrm{~N}$ was shown in the Fig 6 . The descending velocity cycle is $0.01 \mathrm{~s}$. The velocity control frequency is stability. According to the research result of the simulation data, we can know that the average velocity is $1.63 \mathrm{~m} / \mathrm{s}$. The biomedical engineering ruled the safe velocity in landing state is less than $6 \mathrm{~m} / \mathrm{s}$. The simulation result provides the basis for the device structure optimization design. The simulation proved further the feasibility of the escapement velocity control mechanism.

\section{Conclusion}

This paper explains the application situation and development status of the tower circular escape apparatus based on the principle of escapement mechanism. The structure of the working units on the escape apparatus was designed based on the existing mechanism. The commercial virtual prototype software ADAMS was used to simulate the escapement velocity control mechanism. The tall building environment test was finished with the material object simulated prototype. The experiment results show the velocity of the device controlled in the security range. The main mechanism meet the requirements of the strength. The research proved that the development of the tower circular escape apparatus is of great significance to the development and advancement of society in china.

\section{References}

[1] Y. Fu, R. Du, A Study on the Dynamics of Swiss Lever Escapement Mechanism, International Symposium on Collaborative Research in Applied Science, Vancouver, Canada, 2005, pp. 8-15.

[2] A.V. Roup, D.S. Bernstein, On the dynamics of the escapement mechanism of a mechanical clock, Proceedings of the 38th IEEE Conference on Decision and Control, Vol. 3, Phoenix, Arizona, USA, December 1999, pp. 2599-2604.

[3] V. Roup, D.S. Bernstein, S.G. Nersesov, Limit cycle analysis of the verge and foliot clock escapement using impulsive differential equations and Poincare maps, Proceedings of the 2001 American Control Conference, Vol. 4, Arlington, VA, USA, June 2001, pp. 3245-3250.

[4] E.N. Parviz, Computer-Aided Analysis of Mechanical System, Prentice-Hall International Edition, 1998, pp. 42-46.

[5] <http://www.timezone.com/library/horologium/> 